\title{
IVIM Magnetic Resonance Imaging in Guiding Target Delineation and Dose Escalation of Concurrent Radiochemotherapy in Recurrent Stage IIIB Pulmonary Adenosquamous Carcinoma: A Case Report
}

\section{Peng Xu}

Nanchang University Second Affiliated Hospital

\section{Yixuan Chen}

Nanchang University

Anwen Liu

Nanchang University Second Affiliated Hospital

Jing Cai ( $\nabla$ cjdl879@163.com )

the second Affiliated Hospital of Nanchang University https://orcid.org/0000-0002-6792-4959

\section{Case report}

Keywords: NSCLC, functional magnetic resonance, intravoxel incoherent motion, radical concurrent chemoradiotherapy, dose escalation

Posted Date: July 6th, 2020

DOI: https://doi.org/10.21203/rs.3.rs-38927/v1

License: (c) (1) This work is licensed under a Creative Commons Attribution 4.0 International License. Read Full License 


\section{Abstract}

Background: Concurrent radiochemotherapy is regarded as a standard treatment for inoperable, locally advanced non-small cell lung cancer (NSCLC). However, the local control rate is less than $50 \%$. Functional imaging can reveal changes in the tumour microstructure and tumour volume early during the process of radiotherapy, which is beneficial for the early evaluation of efficacy and dose escalation radiotherapy.

Case presentation: Here, we describe a case of recurrent stage IIIB pulmonary adenosquamous carcinoma. Intravoxel incoherent motion (IVIM) was used to guide the delineation of the target area and dose escalation of radical concurrent chemoradiotherapy, and complete remission was achieved.

Conclusion: Our case report provides a novel method for IVIM functional MRI in guiding accurate target delineation and dose escalation of radical radiotherapy.

\section{Background}

Lung cancer is the most common malignant tumour and is harmful to the health of humans worldwide. Approximately $85 \%$ of lung cancers are non-small cell lung cancer (NSCLC), and nearly one-third of NSCLC patients are locally advanced at the consultation and are not suitable for surgical resection. Concurrent radiochemotherapy is regarded as the standard treatment for locally advanced NSCLC [1, 2], but the therapeutic effect is poor, mainly because the toxicity of surrounding normal organs limits the dose of the radiotherapy target[3]. Studies have indicated that a high biological dose and short treatment time are proportional to efficacy and survival [4]. How to use the existing technical measures to increase the target dose without prolonging the total treatment time while decreasing the radiotoxicity of normal organs is the focus of current research. The current study focused on how to improve the curative effect of radical concurrent radiochemotherapy in patients with locally advanced NSCLC by analysing functional image-guided radiotherapy target delineation, that is, the biological target volume (BTV). Functional imaging can reflect alterations in the tumour microstructure and tumour volume early in the process of radiotherapy, which is beneficial for the early evaluation of efficacy and dose escalation radiotherapy. Intravoxel incoherent motion (IVIM) refers to various forms of motion of water molecules in living organisms. IVIM includes intracellular and extracellular free diffusion movement and transcellular membrane movement, which are collectively referred to as slow diffusion movement, and the related movements of microcirculation vascular perfusion are collectively referred to as rapid diffusion movement. It is applied to a series of $b$ values from small to large and fitted to the attenuation of the diffusion-weighted imaging (DWI) signal strength. The slow diffusion motion coefficient (D) refers to the movement of water molecules between cells, representing dispersion; the fast diffusion motion coefficient $\left(D^{*}\right)$ refers to the pseudo-diffusion effect produced by the flow of water molecules in blood vessels, representing perfusion; and the microcirculation perfusion fraction (f) reflects the blood volume, that is, the volume of water protons in blood vessels, representing the volume fraction of rapid diffusion movement. 


\section{Case Presentation}

We report a 57-year-old male non-smoker who underwent "thoracoscopic radical resection of right lower lung cancer (single-hole method)" under general anaesthesia in December 2014. Postoperative pathology showed right lower lung adenosquamous carcinoma (squamous cell carcinoma accounted for $70 \%$, and adenocarcinoma accounted for $30 \%$ ). Nodules with a diameter of $2 \mathrm{~cm}$ were observed at a distance of $4 \mathrm{~cm}$ from the bronchial margin. No cancer was involved in the bronchial margin. Metastatic carcinoma was found in 1 para-bronchial lymph node. The postoperative stage was pT1bN1M0. Human epidermal growth factor receptor (EGFR) gene mutation detection showed 20-ins. Four cycles of NP (vinorelbine + cisplatin) adjuvant chemotherapy were performed. In June 2017, a chest computed tomography (CT) plain scan and an enhanced scan showed a mass shadow of the right upper lobe and segmental atelectasis of the right upper lobe. Recurrence was considered, and enlarged lymph nodes were seen below the carina. The histopathologic findings of the fibreoptic bronchoscopy biopsy demonstrated an invasive carcinoma of the right main bronchus. Abdominal CT, brain MRI and whole-body bone imaging (emission computed tomography; ECT) showed no signs of distant metastasis. The diagnosis was recurrence of lung cancer, rT2N2M0, stage IIIB. Radical concurrent radiochemotherapy was performed under the guidance of IVIM functional MRI.

The radiotherapy regimen was as follows. A Versa HD medical linear accelerator was used, and a 4D CT scanner was used. The upper boundary of the scanning range was cricoid cartilage, and the lower boundary was lumbar 2 cone. The thickness of the scanning layer was $5 \mathrm{~mm}$, and the thickness of the tumour area was $2.5 \mathrm{~mm}$. A GE 3.0T superconducting magnetic resonance scanner (GE Signa HDxt 3.0T MR, GE Corporation), 16-channel abdominal phased array coil, and GE AW4.6 workstation were used. The MRI scan sequences included the T2WI lipid pressure in the transverse position, the T2WI lipid pressure sequence in the coronary position and the IVIM. The MADC image post-processing software of the FuncTool software package was used to obtain three kinds of parameterized images: slow apparent diffusion coefficient (ADC), $D$ * (fast $A D C$ ) and $f$ of fast ADC. Diffusion coefficient ADC images were obtained by conventional DWI post-processing (Fig. 1). Based on the results of the chest CT enhanced scans and IVIM imaging, the tumour tissue in the lung was delineated as the gross tumour volume (GTV), and the subcarinal lymph node was delineated as the GTV of the cervical lymph node (GTVnd). The clinical target volume (CTV) was placed $0.8 \mathrm{~cm}$ outside the GTV. The planned target volume (PTV) was expanded uniformly according to the positioning error of the unit (approximately $0.5 \mathrm{~cm}$ ). The P-GTV dose was 66 Gy/30 F/6 W, and the P-CTV dose was 60 Gy/30 F/6 W (Fig. 2A). After 4 weeks of radiotherapy, the same scanning and post-processing methods were used to review the results after radiotherapy (Fig. 1), and the GTV and CTV were re-drawn according to the post-treatment images. Changes in tumour size and in various parameters were observed. According to the IVIM images before and after treatment, another tumour treatment resistance area was outlined and named the GTV-r. The dose of the GTV-r was increased, and the physical dose of radiotherapy reached $44 \mathrm{~Gy} / 20 \mathrm{~F}+24 \mathrm{~Gy} / 10 \mathrm{~F}$ (the biological dose was $53.7 \mathrm{~Gy}+29.8 \mathrm{~Gy}=83.5 \mathrm{~Gy}$ ) (Fig. 2B). The concurrent chemotherapy regimen was weekly docetaxel combined with cisplatin. One month after treatment, chest and abdominal CT and cranial MRI were performed again. The mass shadow of the right hilar area had disappeared, and the 
upper lobe of the right lung had re-expanded. The curative effect was evaluated as complete remission (Fig. 3). Grade 2 radiation pneumonitis occurred during treatment and returned to normal after treatment. After regular re-examination, the patient's last follow-up was April 17,2020, and there were no signs of recurrence or metastasis. The patient's performance status (PS) score was 0 points.

\section{Discussion And Conclusions}

Concurrent radiochemotherapy is still the standard treatment for inoperable, locally advanced NSCLC. However, the local control rate is less than $50 \%$, and the 5-year survival rate is only approximately $10-$ $15 \%$ [3]. The main reason for the poor treatment effect is local failure, and the main reason for local failure is the unreasonable design of the target area. In terms of target location, functional imaging can account for the deficiencies in conventional CT. According to research reports, the target area of radical concurrent chemoradiotherapy delineated based on PET/CT in NSCLC patients is smaller than that delineated based on conventional CT, which is conducive to a dose increase (66.6 $73.8 \mathrm{~Gy})$, and the recurrence rate in the field is low [5]. Yang[6] showed that DW-MRI can be used as an alternative to PET/CT for precise positioning of the target area. Tang [7] reported that dynamic contrast-enhanced (DCE)-MRI is superior to 64-MDCT in distinguishing NSCLC stages T3 and T4. Schaarschmidt [8] reported that DW-MRI has high specificity (>90\%) in the discrimination of NSCLC lymph node metastasis, and the $A D C$ value is inversely correlated with the standardized uptake value (SUV) of PET/CT. Both can be used to identify NSCLC lymph node metastasis. Functional images can better reflect changes in the microstructure than anatomical images. For example, Mahasittiwat[9] reported that a reduction in the metabolic tumour volume (MTV) was significantly larger than that derived from ordinary CT when NSCLC was irradiated with a 2/3 radical dose, which indicated that the dose to the target area could be increased by using functional imaging to guide radiotherapy. Kong[10] proposed a significant change in tumour size and the SUV when the radiation dose reached $45 \mathrm{~Gy}$. When the radiation dose reaches $45 \mathrm{~Gy}$, incremental radiotherapy with a reduced field can increase the radiation dose in the radiation resistance area to the tumour while reducing or not increasing the radiation dose to normal tissue. The ongoing study of RTOG1106 takes advantage of this idea and adjusts the target area according to PET/CT during radiotherapy to increase the dose to the tumour to $80.4 \mathrm{~Gy}$ [10]. Regarding the problem associated with functional imaging, local recurrence can be detected in a timely manner. P Munoz Schuffenegger[11] described 30 patients who were treated with lung stereotactic body radiation therapy (SBRT),and MRI was used to diagnose seven patients with local recurrence, which was later confirmed in three patients; moreover, MRI did not miss any case of true local recurrence. The MRI protocol incorporating DWI and DCE is feasible and can confirm the suspicion of local recurrence in patients with highly suspicious CT scans.

Here, we present a patient with recurrent stage IIIB lung adenosquamous carcinoma after surgery who was guided by IVIM functional MRI to delineate the target area of concurrent chemoradiotherapy, and the field reduction dose was increased during radiotherapy. After the biological dose of radiotherapy reached 83.5 Gy, the efficacy was completely relieved. To date, the last follow-up was on April 17, 2020, and no recurrence or metastasis was observed in the imaging results. 


\section{Abbreviations}

NSCLC: Non-small cell lung cancer; IVIM: Intravoxel incoherent motion; BTV: Biological target volume; MRI: Magnetic resonance imaging; EGFR: Epidermal growth factor receptor; ECT: Emission computed tomography; CT: Computed tomography; GTV: Gross target volume; CTV: Clinical target volume; PTV: Planned target volume; MTV: Metabolic tumour volume; ADC: Apparent diffusion coefficient; DWI: Diffusion-weighted imaging; SUV: Standardized uptake value; SBRT: Stereotactic body radiation therapy.

\section{Declarations}

\section{Ethics approval and consent to participate}

This research was deemed ethical by our institution's Ethics approval and the consent about the publication of this case was given by the participate.

\section{Availability of data and materials}

The datasets used and analysed during the current study are available from the corresponding author on reasonable request.

\section{Competing interests}

The authors declare that they have no competing interests.

\section{Funding}

This study was supported by the National Natural Science Foundation of China (Project label: 81760566) and the Natural Science Foundation of Jiangxi Province, China (Project label: 20161BBG70210).

\section{Authors' contributions}

Jing Cai collected patient data, composed the manuscript, and produced the final draft. Peng Xu and Yixuan Chen assisted in composing drafts of the manuscript and edited the manuscript. Anwen Liu provided revisions for the manuscript. All authors read and approved the final manuscript.

\section{Acknowledgements}

We have obtained permission to acknowledge all those mentioned in the Acknowledgements section. 


\section{References}

1. Nakamichi S, Horinouchi H, Asao T, Goto Y, Kanda S, Fujiwara Y et al.Comparison of Radiotherapy and Chemoradiotherapy for Locoregional Recurrence of Non-small-cell Lung Cancer Developing After Surgery. Clin Lung Cancer. 2017, 18:e441-e448.

2. Ishihara M, Igawa S, Sasaki J, Otani S, Fukui T, Ryuge S et al.Evaluation of concurrent chemoradiotherapy for locally advanced NSCLC according to EGFR mutation status. Oncol Lett. 2017, 14:885-890.

3. Brown T, Pilkington G, Boland A, Oyee J, Tudur Smith C, Dundar Y et al.Clinical effectiveness of firstline chemoradiation for adult patients with locally advanced non-small cell lung cancer: a systematic review. Health Technol Assess. 2013, 17:1-99.

4. Machtay M, Bae K, Movsas B, Paulus R, Gore EM, Komaki R et al.Higher biologically effective dose of radiotherapy is associated with improved outcomes for locally advanced non-small cell lung carcinoma treated with chemoradiation: an analysis of the Radiation Therapy Oncology Group. Int $J$ Radiat Oncol Biol Phys. 2012, 82:425-434.

5. Zheng $Y$, Sun $X$, Wang J, Zhang L, Di X, Xu Y.FDG-PET/CT imaging for tumor staging and definition of tumor volumes in radiation treatment planning in non-small cell lung cancer. Oncol Lett. 2014, 7:1015-1020.

6. Yang RM, Li L, Wei XH, Guo YM, Huang YH, Lai LS et al.Differentiation of central lung cancer from atelectasis: comparison of diffusion-weighted MRI with PET/CT. PLoS One. 2013, 8:e60279.

7. Tang W, Wu N, OuYang H, Huang Y, Liu L, Li M.The presurgical T staging of non-small cell lung cancer: efficacy comparison of 64-MDCT and 3.0 T MRI. Cancer Imaging. 2015, 15:14.

8. Benedikt Michael Schaarschmidt, Christian Buchbender, Felix Nensa, Johannes Grueneisen, Benedikt Gomez, Jens Köhler et al.Correlation of the Apparent Diffusion Coefficient (ADC) With the Standardized Uptake Value (SUV) in Lymph Node Metastases of Non-Small Cell Lung Cancer (NSCLC) Patients Using Hybrid 18F-FDG PET/MRI. PLoS One. 2015, 10:e0116277.

9. Mahasittiwat P, Yuan S, Xie C, Ritter T, Cao Y, Ten Haken RK et al.Metabolic Tumor Volume on PET Reduced More than Gross Tumor Volume on CT during Radiotherapy in Patients with Non-Small Cell Lung Cancer Treated with 3DCRT or SBRT. J Radiat Oncol. 2013, 2:191-202.

10. Kong FM, Zhao J, Wang J, Faivre-Finn C.Radiation dose effect in locally advanced non-small cell lung cancer. J Thorac Dis. 2014, 6:336-347.

11. P Munoz-Schuffenegger, S Kandel , Z Alibhai , A Hope, A Bezjak , A Sun et al.A Prospective Study of Magnetic Resonance Imaging Assessment of Post-radiation Changes Following Stereotactic Body Radiation Therapy for Non-small Cell Lung Cancer. Clin Oncol (R Coll Radiol). 2019, 31:720-727.

\section{Figures}




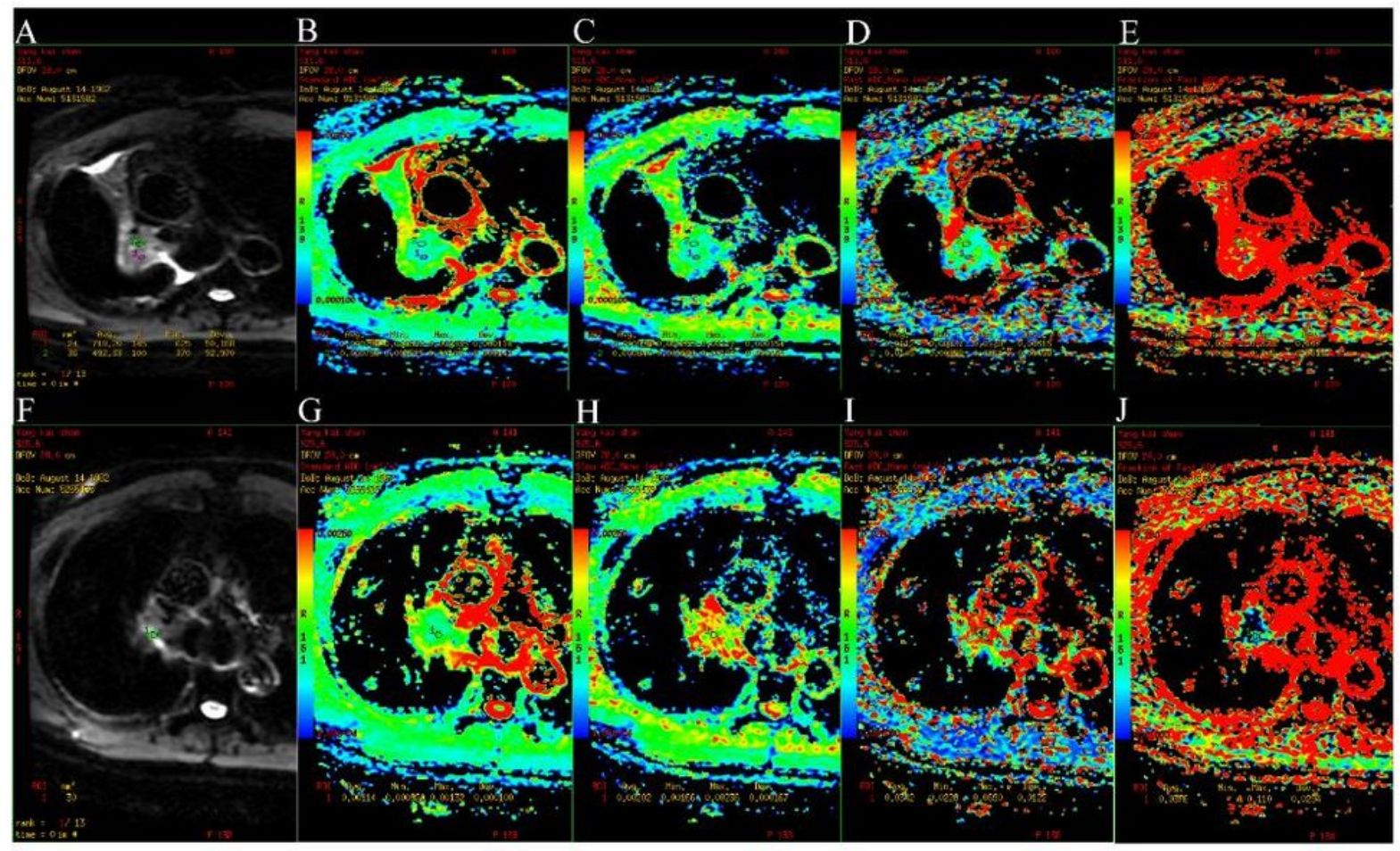

Figure 1 IVIM images before and after radiotherapy, A-E: before radiotherapy,F-J: 4 weeks after radiotherapy. A and F: MRI plain scan, B and G: diffusion coefficient ADC images, $\mathrm{C}$ and H: slow diffusion coefficient, D and I: fast diffusion coefficient, $\mathrm{E}$ and $\mathrm{J}$ : volume fraction $\mathrm{f}$ of fast diffusion.

\section{Figure 1}

Figure 1

A
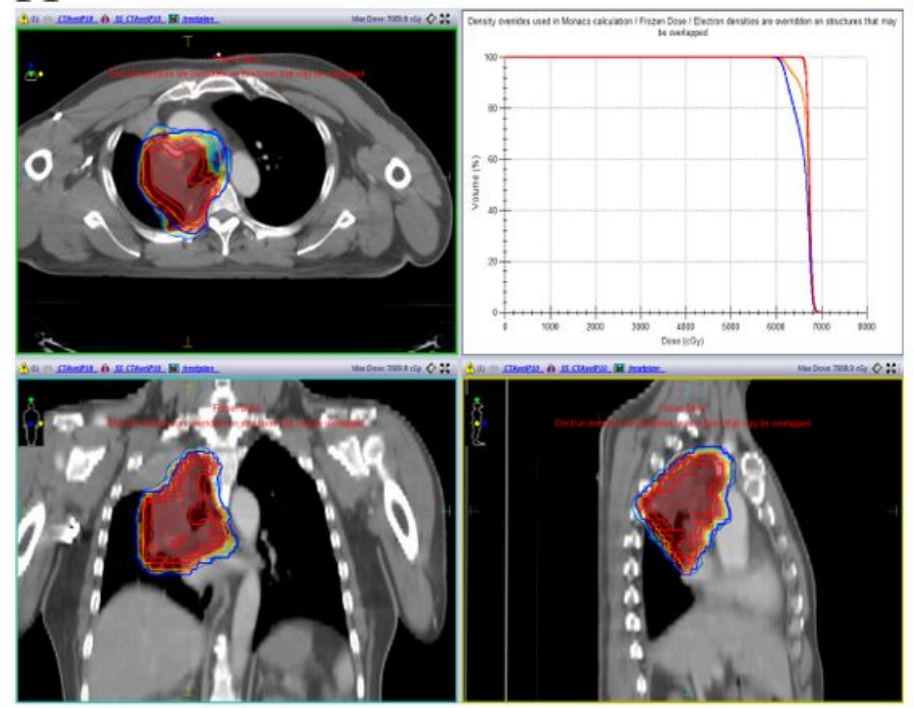

B

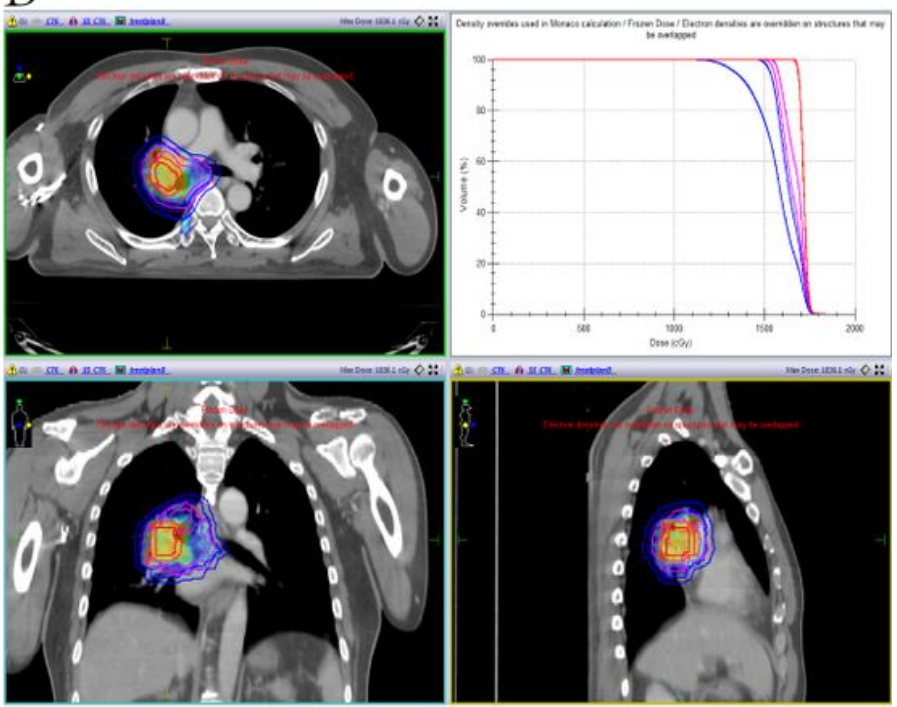

Figure 2 Radiation treatment planning. A: Initial radiotherapy plan, B: Dose escalation plan after 4 weeks of initial radiotherapy

\section{Figure 2}

Figure 2 


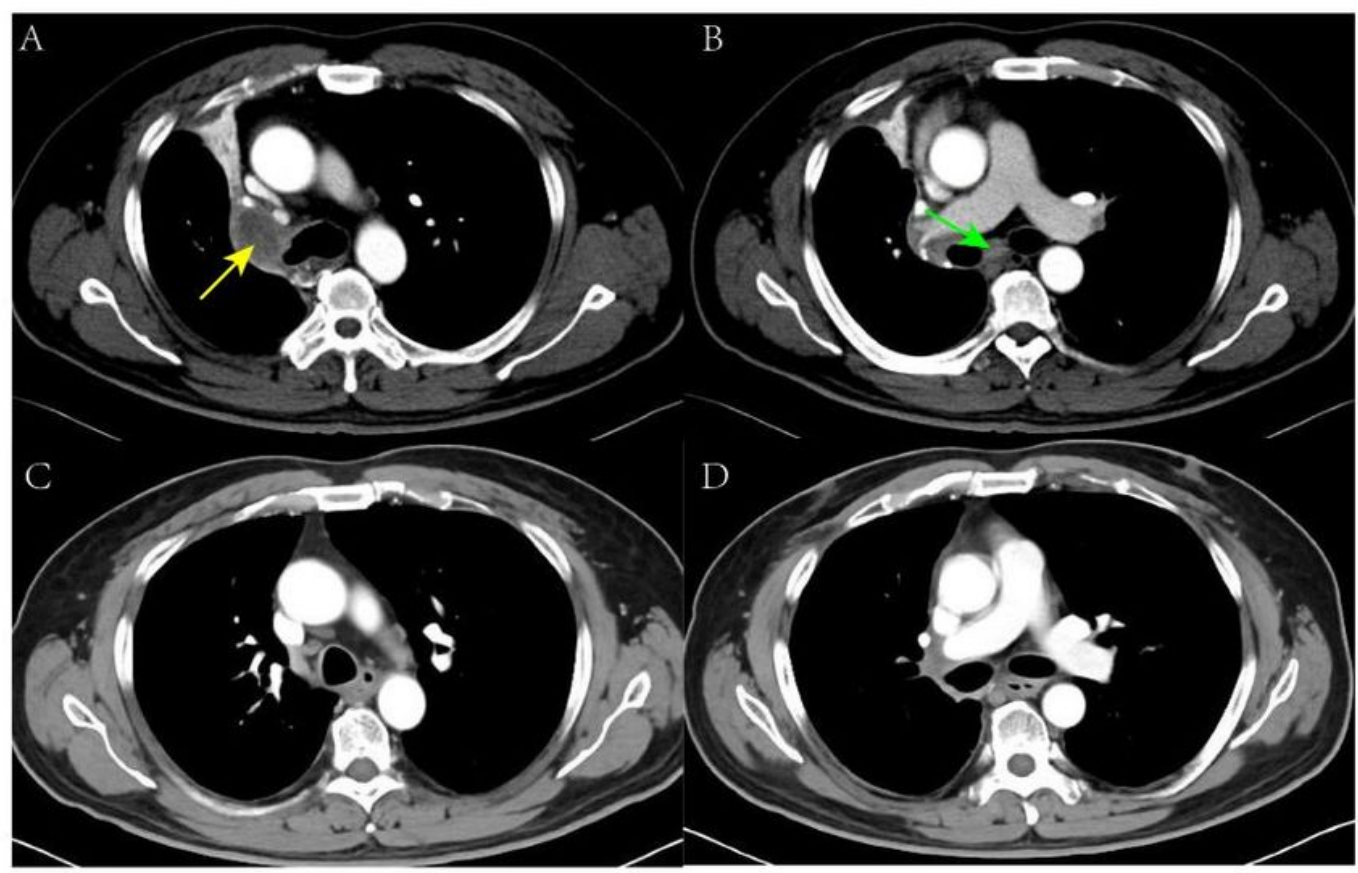

Figure 3 Chest CT before and after chemoradiotherapy. A: right hilar occupying before radiochemotherapy (Yellow arrow : tumor before radiochemotherapy) ; B: subcarinal enlarged lymph nodes before radiochemotherapy (Green arrow: lymph node before radiochemotherapy) ; C: right hilar occupying disappeared after radiochemotherapy; D: subcarinal enlarged lymph nodes disappeared after radiochemotherapy.

\section{Figure 3}

Figure 3 